\title{
Efeitos de diferentes níveis de cálcio dietético na cinética de cálcio e fósforo em eqüinos ${ }^{1}$
}

\section{Dorinha Miriam Silber Schmidt Vitti ${ }^{2}$, Carlos Eduardo Furtado ${ }^{3}$, João Batista da Silva Quadros ${ }^{3}$, João Batista Lopes ${ }^{4}$, Ives Cláudio da Silva Bueno², Eduardo Fernando Nozella², Patrícia Barboza de Godoy ${ }^{2}$}

\author{
1 Pesquisa financiada pela FAPESP - projeto $n^{\circ}$ 05/14532-5 e pelo CNPq n 470059/2004-4 \\ ${ }^{2}$ Centro de Energia Nuclear na Agricultura, Caixa Postal 96, CEP: 13400-970, Piracicaba - SP, Brasil. \\ ${ }^{3}$ Universidade Estadual de Maringá. \\ 4 Universidade Federal do Piauí, Campus da Socopo, Teresina, PI.
}

RESUMO - Objetivou-se estudar o metabolismo de cálcio $(\mathrm{Ca})$ e fósforo $(\mathrm{P})$ nos eqüinos em crescimento que receberam diferentes níveis de suplementação de $\mathrm{Ca}: 0,15 ; 0,45$ e $0,75 \%$ na dieta, utilizando-se o modelo determinístico e compartimental. Foram utilizadas informações sobre o estudo de metabolismo e cinética de Ca e P em tecidos, obtidas pela técnica da diluição isotópica. Constatou-se que os níveis dietéticos de Ca tiveram influência na absorção real do Ca, sendo menor para o nível $0,15 \%$ (4,97 $\mathrm{g} \mathrm{Ca} / \mathrm{dia})$. As trocas de $\mathrm{Ca}$ entre o sangue e o trato digestivo foram menores para o nível $0,15 \%$. A mobilização entre o sangue e os ossos e sangue e tecidos moles não foi influenciada pelos tratamentos, mas o balanço nos ossos e tecidos foi menor para o nível $0,15 \%$. Os níveis de Ca dietéticos tiveram influência no $\mathrm{P}$ eliminado através da urina, sendo este valor maior para o tratamento $0,15 \%(2,49 \mathrm{~g} /$ animal/dia $)$. A absorção real média do $\mathrm{P}$ fo de $83 \%$, não havendo diferenças para os tratamentos. Verificou-se deposição óssea média de $9,69 \mathrm{~g} \mathrm{P} / \mathrm{animal} / \mathrm{dia}$, indicando que a quantidade de $\mathrm{P}$ fornecida foi adequada em relação à tolerância permissível à amplitude na relação Ca:P para a espécie na categoria animal estudada. Não houve diferenças significativas entre os fluxos de $\mathrm{P}$ nos diversos compartimentos, por intermédio do modelo de metabolismo. A ingestão de níveis crescentes de $\mathrm{Ca}$ afetou o metabolismo e a cinética deste elemento, entretanto, a proporção Ca:P é o fator predominante para determinar a excreção, retenção e absorção de Ca A deposição de $\mathrm{Ca}$ no osso foi influenciada pela quantidade ingerida deste mineral. O metabolismo de $\mathrm{P}$ em eqüinos em crescimento não foi afetado pelos teores de Ca. O fornecimento de $13,4 \mathrm{~g} \mathrm{P} / \mathrm{animal} / \mathrm{dia}$ foi suficiente para manter o metabolismo de $\mathrm{P}$ nos padrões considerados normais.

Palavras-chave: fluxo de minerais, macrominerais, potros, radioisótopos

\section{Effects of different levels of dietary calcium on calcium and phosphorus kinetics in horses}

\begin{abstract}
This experiment was carried out to study calcium $(\mathrm{Ca})$ and phosphorus $(\mathrm{P})$ kinetics of growing horses fed diets with different Ca levels: $0.15 \%, 0.45$, and $0.75 \%$, using a deterministic and compartmental model. The information on metabolism and kinetic of $\mathrm{Ca}$ and $\mathrm{P}$ in tissues was obtained by the isotopic dilution technique. Dietary Ca levels influenced $\mathrm{Ca}$ true absorption, showing lower value $(4.97 \mathrm{~g} \mathrm{Ca} / \mathrm{day})$ for the Ca level of $0.15 \%$. The changes of Ca between the blood and digestive tract were lower for $0,15 \% \mathrm{Ca}$. The flows between the blood and bone and blood and soft tissues did not show differences between treatments, but bone and tissue balance was lower for the level of $0.15 \%$. Dietary Ca levels influenced on $\mathrm{P}$ in urine and values were higher for $0.15 \%$ (2.49 g P/anim.day). The mean true $\mathrm{P}$ absorption was of $83 \%$, with no differences among treatments. Bone deposition of $9.69 \mathrm{~g}$ P/anim.day was observed, showing that the amount of P fed was adequate in relation to the allowed amplitude for Ca:P ratio for the specie in the studied animal category. No differences between the $\mathrm{P}$ flows in the diverse compartments, for interference of the metabolism model. The ingestion of increasing levels of $\mathrm{Ca}$ affected the metabolism and the kinetic of this element, however $\mathrm{Ca}: \mathrm{P}$ ratio is the predominant factor to determine the excretion, retention and absorption of $\mathrm{Ca}$. The $\mathrm{Ca}$ deposition in bone is influenced by the amount ingested of this mineral. The P metabolism in growing horses was not affected by Ca levels. The amount of $13.4 \mathrm{~g}$ P/anim.day in the diet was sufficient to maintain $\mathrm{P}$ metabolism at normal standard levels.
\end{abstract}

Key Words: mineral flow, macromineral, foals, radioisotope 


\section{Introdução}

A deficiência de cálcio ( $\mathrm{Ca}$ ) nos eqüinos é um importante fator que influi na produtividade. Os mecanismos básicos do metabolismo de Ca diferem substancialmente entre as espécies; nos eqüinos, sua regulação é menos estudada e entendida que a das outras espécies.

Nos eqüinos, as exigências minerais são complexas, podendo ocorrer interações de minerais durante os processos de digestão e absorção. Um exemplo desta complexidade é a interação $\mathrm{Ca} \times \mathrm{P}$. Altas concentrações de $\mathrm{Ca}$ podem acarretar deficiência de $\mathrm{P}$, mesmo que o consumo de $\mathrm{P}$ seja adequado. Há uma estreita relação metabólica entre o Ca e P (Tamin \& Angel, 2003), que estão presentes principalmente nos ossos, na forma de hidroxiapatita, e são mobilizados quando as exigências dos animais não são alcançadas. Chapuis-Lardy et al. (2004) sugeriram que a concentração de $\mathrm{Ca}$ afeta a excreção de $\mathrm{P}$ fecal, devido à formação de complexo Ca-P que reduz a disponibilidade de ambos os minerais.

Os estudos físico-químicos sobre o metabolismo nos ossos mostram que as trocas de $\mathrm{Ca}$ entre os ossos e fluidos corporais ocorrem por dois processos: a) trocas iônicas, que correspondem ao processo rápido, na superfície óssea, quando excesso de Ca é incorporado à molécula de fosfato tricálcico; b) trocas lentas ou processo de recristalização, que correspondem à penetração de Ca trocável no interior do osso.

A intensidade desses processos não pode ser medida por métodos clássicos. $\mathrm{O}$ uso de $\mathrm{Ca}$ radioativo permite estudar aspectos dinâmicos do metabolismo desse mineral. A técnica de diluição isotópica tem sido aplicada a estudos de metabolismo de Ca e P, especialmente em ruminantes (Salviano \& Vitti, 1998), mas poucas informações existem com relação ao $\mathrm{Ca}$ em eqüinos. Pela técnica com radioisótopos, é possível obter informações da absorção real de minerais, que não são verificadas com as técnicas tradicionais de balanço. Os dados obtidos por esta metodologia, aplicados a modelos matemáticos, têm trazido esclarecimentos importantes com relação à cinética de $\mathrm{Ca} \mathrm{e}$ $\mathrm{P}$ à determinação das exigências nutricionais dos animais.

O metabolismo e a exigência de Ca são de grande interesse na indústria de eqüinos, especialmente com relação aos animais em crescimento. Para esta categoria animal, as exigências são estimadas com pôneis (Schryver et al., 1970).

Vitti et al. (2000), utilizando dados de experimentos de balanço e cinética de $\mathrm{P}$ em caprinos, propuseram modelo matemático contendo quatro compartimentos (trato, sangue, ossos e tecidos moles) para o estudo do efeito de níveis crescentes de P nos animais em crescimento. Este modelo foi recentemente revisado (Dias et al., 2006) para o estudo da cinética de $\mathrm{P}$ em caprinos e da cinética de $\mathrm{Ca}$ em ovinos.

Os resultados das pesquisas com $\mathrm{P}$ e Ca têm apresentado grande variação, decorrente não apenas do crescente desenvolvimento e produtividade animal, mas também de importantes avanços na tecnologia da nutrição e dos sistemas de criação, especialmente quando se trata de produção em grande escala. Por outro lado, a indústria de alimentos necessita de informações precisas para produzir rações que atendam às exigências dos animais com menor custo (Cupák et al., 1972), uma vez que tem sido demonstrado que níveis adequados de mineral para crescimento são insuficientes para a máxima mineralização (Cromwell, 1991).

O objetivo neste trabalho foi validar, por meio de modelos matemáticos, a cinética de $\mathrm{Ca}$ e $\mathrm{P}$ em eqüinos em crescimento recebendo dietas suplementadas com diferentes níveis de Ca.

\section{Material e Métodos}

O experimento foi conduzido na Fazenda e Haras Braido, município de Cerqueira César (SP) e no Laboratório de Nutrição Animal do Centro de Energia Nuclear na Agricultura (CENA/USP), Piracicaba, São Paulo. Foram utilizados 12 eqüinos machos com idade média de dez meses e peso médio de $221,0 \pm 69.99 \mathrm{~kg}$.

Os tratamentos consistiram de três dietas, compostas por níveis crescentes de cálcio (baixo $=0,15 \%$; padrão $=$ $0,45 \%$; alto $=0,75 \%)$, e nível padrão de fósforo $(0,23 \%)$ (Tabela 2). Para obter os níveis crescentes de cálcio, as dietas (nível padrão e alto de C) foram suplementadas com núcleo mineral (Potensal ${ }^{\circledR}$ ) (Tabelas 1 e 2).

As normas de alimentação foram realizadas de acordo com as recomendações do NRC (1989) e Ott (1995): os animais receberam as dietas na razão de $2,6 \%$ do peso vivo/ $\mathrm{kg} / \mathrm{animal} / \mathrm{dia}$ ( $60 \%$ de concentrado e $40 \%$ de feno Tifton 85 ), correspondendo a um consumo médio de $6,23 \mathrm{~kg}$ alimento/ animal/dia. As dietas foram oferecidas em três refeições diárias, às 8,13 e $18 \mathrm{~h}$, sendo o concentrado (contendo o núcleo mineral) fracionado em partes iguais e $2 / 3$ do feno oferecido na última refeição.

A análise químico-energética das dietas foi realizada segundo as recomendações da AOAC (1980). Os conteúdos de Ca e P foram analisados após determinação das cinzas e de sua digestão em ácido clorídrico concentrado. A determinação do P inorgânico foi feita por colorimetria, usando-se o método vanadato-molibdato (Sarruge \& Haag, 1974), e a do 
Tabela 1 - Composição química dos ingredientes das dietas experimentais (base na matéria seca - MS\%)

\begin{tabular}{lccc}
\hline $\begin{array}{l}\text { Composição químical } \\
\text { Ingrediente }\end{array}$ & Milho & $\begin{array}{c}\text { Farelo de } \\
\text { soja }\end{array}$ & $\begin{array}{c}\text { Feno de } \\
\text { Tifton } 85\end{array}$ \\
\hline MS (\%) & 89,77 & 89,31 & 91,73 \\
PB (\%) & 9,78 & 42,48 & 7,67 \\
EB (kcal/kg) & $4.458,48$ & $4.545,95$ & $4.181,35$ \\
Ca (\%) & 0,05 & 0,35 & 0,26 \\
P (\%) & 0,27 & 0,63 & 0,12 \\
FDN (\%) & 9,36 & 17,77 & 84,55 \\
FDA (\%) & 2,75 & 11,57 & 41,37 \\
EE (\%) & 4,14 & 2,17 & 2,06 \\
MM (\%) & n.d & n.d & 5,26 \\
Lignina (\%) & n.d & n.d & 11,78 \\
\hline
\end{tabular}

n.d. não determinado.

Tabela 2 - Composição percentual de ingrediente e nutricional das dietas experimentais (\%MS)

\begin{tabular}{lrrr}
\hline & \multicolumn{3}{c}{ Nível de Ca (\%) } \\
\cline { 2 - 4 } Ingrediente (\%) & $0,15^{1}$ & $0,45^{2}$ & $0,75^{3}$ \\
\hline Milho - grãos moídos & 48,56 & 47,56 & 46,56 \\
Farelo de soja & 10,84 & 11,14 & 11,34 \\
Feno de Tifton 85 & 40,00 & 40,00 & 40,00 \\
Núcleo mineral & 0,50 & 1,30 & 2,10 \\
\hline Composição nutricional & & & \\
e calculada & & & \\
\hline MS (\%) & 90,59 & 90,89 & 91,02 \\
PB (\%) & 11,87 & 11,90 & 11,72 \\
Ca (\%) & 0,15 & 0,45 & 0,75 \\
P (\%) & 0,23 & 0,23 & 0,23 \\
FDN (\%) & 49,72 & 50,97 & 50,95 \\
FDA (\%) & 19,52 & 19,33 & 19,58 \\
EE (\%) & 3,14 & 3,12 & 3,12 \\
MM (\%) & 3,82 & 4,24 & 4,76 \\
Composição calculada & & & \\
ED (Mcal/kg) & 2,68 & 2,68 & 2,68 \\
Lisina (\%) & 0,47 & 0,47 & 0,47 \\
\hline
\end{tabular}

${ }^{1}$ Núcleo mineral (kg/produto): cobre $-2100 \mathrm{mg}$, iodo $-20 \mathrm{mg}$, manganês $-8.000 \mathrm{mg}$, selênio - $20 \mathrm{mg}$, zinco $-8.000 \mathrm{mg}$, cobalto $-20 \mathrm{mg}$, enxofre $-10,31 \%$, magnésio $-10,42 \%$ e sódio $-25 \%$.

2 Núcleo mineral (kg/produto): cobre $-800 \mathrm{mg}$, iodo $-8 \mathrm{mg}$, manganês $3.000 \mathrm{mg}$, selênio $-8 \mathrm{mg}$, zinco $-3.000 \mathrm{mg}$, cobalto $-8 \mathrm{mg}$, enxofre $3,84,31 \%$, magnésio - 3,85\%, sódio - 9,23\% e cálcio - $23 \%$.

3 Núcleo mineral (kg/produto): cobre $-500 \mathrm{mg}$, iodo $-5 \mathrm{mg}$, manganês $1.900 \mathrm{mg}$, selênio $-5 \mathrm{mg}$, zinco $-1.900 \mathrm{mg}$, cobalto $-5 \mathrm{mg}$, enxofre $2,38 \%$, magnésio - 2,38\%, sódio - 5,71\% e cálcio - $28,6 \%$.

${ }^{4}$ Valores estimados pelo NRC (1989)

Ca, por espectrofotometria de absorção atômica (Zagatto et al., 1979).

Durante o período pré-experimental (10 dias), os animais permaneceram na Fazenda e Haras Braido, onde receberam vermífugo de amplo espectro (0,2 $\mathrm{mg}$ ivermectina $/ \mathrm{kg}$ de peso vivo) e passaram pela fase de adaptação às dietas experimentais, permanecendo em baias individuais de alvenaria semi-abertas $\left(25 \mathrm{~m}^{2}\right)$ com piso de cimento, sem cama e bebedouro de cimento.

Durante o período experimental (sete dias), os animais permaneceram no biotério do laboratório de Nutrição
Animal/CENA, em construção de alvenaria, com área de aproximadamente $200 \mathrm{~m}^{2}$. Nesse período, os animais permaneceram em confinamento total, em gaiolas para ensaios metabólicos, segundo modelo adaptado de Furtado et al. (2000), onde receberam as dietas experimentais e água para consumo à vontade.

Na seqüência, foram coletados aproximadamente $200 \mathrm{~g}$ de fezes de cada animal, para exame parasitológico, e plasma, fezes e urina, para análises de fósforo e cálcio inorgânicos, antes da injeção do elemento radioativo. Em seguida, foram injetados em cada animal, através da veia jugular direita, 30 MBq (megaBecquerel- unidade de medida da grandeza atividade radioativa) ${ }^{45} \mathrm{Ca}$, como solução de cloreto de cálcio aquoso, e $30 \mathrm{MBq}{ }^{32} \mathrm{P}$, como solução de fosfato de sódio, livres de carregador (Gênese Produtos Farmacêuticos e Diagnósticos). Após a injeção, amostras de sangue foram coletadas através da jugular esquerda, usando-se tubos a vácuo contendo heparina, aos 5 minutos e 24, 48, 72, 96, 120 e 144 horas. Foram realizadas coletas totais diárias de fezes e urina pela manhã, a partir de 24 horas após a injeção do radioisótopo, até o sétimo dia, sendo que uma alíquota de $5 \%$ do total diário excretado foi coletada e armazenada em congelador para posterior análise. O mesmo procedimento das fezes foi adotado para a urina, quando foram coletadas amostras de $1 \%$ do volume total diário.

Após o período de coleta, os eqüinos foram sacrificados de maneira indolor e sem sofrimento, utilizando injeção venosa de $20 \mathrm{~mL}$ de solução de T-61 (Intervet International $\mathrm{GmbH}$ ), composta por iodeto de mebezônio (efeito paralisante na musculatura), embutramida e cloridato de tetracaína (anestésicos). Em seguida, os animais sofreram exsanguinação por jugulação bilateral, sendo então coletadas amostras de tecidos $\left(12^{\mathrm{a}}\right.$ costela, fígado, rim, coração e músculo) para as determinações de $\mathrm{Ca}$ inorgânico e radioativo. As carcaças foram posteriormente levadas para um depósito construído de acordo com normas da Comissão Nacional de Energia Nuclear (CNEN), órgão responsável pelos assuntos relacionados à proteção radiológica no País. Os depósitos foram construídos para que houvesse total blindagem da radiação emitida até o decaimento do material radioativo. Todas as operações com radioisótopos foram supervisionadas pelo Serviço de Proteção Radiológica do CENA.

As amostras de sangue foram centrifugadas a $1.100 \mathrm{~g}$ (3.500 rpm), durante 10 minutos, para obtenção do plasma e posterior determinação do Ca e $\mathrm{P}$ inorgânicos e radioativos. Após a precipitação das proteínas do plasma com ácido tricloroacético, o Ca e $\mathrm{P}$ inorgânicos foram determinados por espectrofotometria de absorção atômica (Zagatto 
et al., 1979) e colorimetria (Fiske \& Subbarow, 1925), respectivamente. Pelo método colorimétrico o fosfato inorgânico presente nas amostras, em meio ácido, forma com o molibdato de amônio, um complexo denominado fosfomolibdato de amônio $\left(\left(\mathrm{NH}_{4}\right)_{3}\left[\mathrm{PO}_{4}\left(\mathrm{MoO}_{3}\right)_{12}\right]\right)$. Este complexo é reduzido pelo ácido aminonaftol sulfônico, resultando em coloração azul, que é comparada, por colorimetria, com solução padrão $(340 \mathrm{~nm})$.

As amostras de fezes $(5 \%$ do total diário coletado de cada animal) foram maceradas manualmente, com auxílio de pistilo, homogeneizadas e secas. Após a determinação das cinzas, a partir de $1 \mathrm{~g}$ de amostra seca, realizaram-se a digestão com $5 \mathrm{~mL}$ de ácido clorídrico concentrado e as leituras do Ca, por meio da espectrofotometria de absorção atômica (Zagatto et al., 1979), e do P, por colorimetria (Fiske \& Subbarow, 1925). Cerca de $5 \mathrm{~g}$ de cada tecido foram incinerados em mufla e, após digestão com ácido sulfúrico 18 N, fez-se a determinação do Ca e P inorgânicos pelos mesmos métodos supracitados.

Para determinação do teor de Ca e P inorgânicos na urina, utilizaram-se $30 \mathrm{~mL}$ da amostra, que foram submetidos à digestão a quente, em $\mathrm{HCl} 12 \mathrm{~N}$, seguida de secagem do material a $55^{\circ} \mathrm{C}$ em estufa e posterior queima a $500^{\circ} \mathrm{C}$. As cinzas obtidas por este processo foram diluídas em $\mathrm{HCl} 3 \mathrm{~N}$, ajustando-se o volume em balão volumétrico de $10 \mathrm{~mL}$ (Morse et al., 1992). O teor de Ca inorgânico foi obtido por meio da espectrofotometria de absorção atômica (Zagatto et al., 1979) e o teor de P, pela colorimetria.

Preparou-se uma solução cintiladora para detecção de ${ }^{45} \mathrm{Ca}$ contendo $4 \mathrm{~g}$ de difeniloxazole (PPO) e $0,3 \mathrm{~g}$ de 1,4 bis(5-feniloxazole-2-il) benzeno (POPOP), dissolvidos em mistura de $500 \mathrm{~mL}$ de triton $\mathrm{X}-100$ e $1 \mathrm{~L}$ de tolueno.

Pipetou-se $1 \mathrm{~mL}$ de amostras de plasma e urina acidificadas com três gotas de $\mathrm{HCl}$ e $10 \mathrm{~mL}$ de solução cintiladora em frascos de borosilicato para cintilação líquida. Solução de cinzas resultantes das fezes (1 mL) e $10 \mathrm{~mL}$ de solução cintiladora foram adicionados em frascos de borosilicato para cintilação líquida. A detecção da radioatividade das amostras foi feita pelo método de cintilação líquida. O equipamento utilizado foi um sistema Beckman LS-5000 TA, equipado com circuito de coincidência, três canais de contagem simultâneos e uma fonte radioativa de $1,11 \mathrm{MBq}{ }^{137} \mathrm{Cs}$, para padronização externa automática.

Para determinação do $\mathrm{P}$ radioativo, as amostras de plasma e urina foram diluídas em água destilada $(1: 20) \mathrm{e}$ a detecção, feita por efeito Cerenkov (IAEA, 1979). Após processamento de cinzas e digestão das amostras de fezes e tecidos, procedeu-se à detecção do $\mathrm{P}$ radioativo, conforme procedimento descrito anteriormente.
Para o estudo da cinética do $\mathrm{Ca}$ e $\mathrm{P}$, foi utilizado o modelo de Vitti et al. (2000) (Figura 1).

As equações diferenciais que descrevem a dinâmica do comportamento do sistema baseiam-se nos princípios de conservação de massa. Assume-se que o sistema está parcialmente em equilíbrio e, assim, as equações são obtidas igualando-as a zero e manipulando matematicamente para se obterem os fluxos de interesse. Os símbolos na Figura 1 são caracterizados pelas equações de 1 a 7 :

$\mathrm{F}_{12}=\mathrm{s}_{1} \tilde{\mathrm{F}}_{10} /\left(\mathrm{s}_{2}-\mathrm{s}_{1}\right)$

$\mathrm{F}_{21}=\tilde{\mathrm{F}}_{10}+\mathrm{F}_{12}-\tilde{\mathrm{F}}_{01}$

$\mathrm{F}_{32}=\mathrm{s}_{3} \mathrm{Q}_{3} /\left[\mathrm{t}\left(\mathrm{s}_{2}-\mathrm{s}_{3}\right)\right]$

$\mathrm{F}_{42}=\mathrm{s}_{4} \mathrm{Q}_{4} /\left[\mathrm{t}\left(\mathrm{s}_{2}-\mathrm{s}_{4}\right)\right]$

$\left|\mathrm{F}_{23}+\mathrm{F}_{24}\right|=\mathrm{F}_{02}+\mathrm{F}_{12}+\mathrm{F}_{32}+\mathrm{F}_{42}-\mathrm{F}_{21}$

$\mathrm{F}_{24}=\left(\mathrm{s}_{3+4}-\mathrm{s}_{3}\right)\left|\mathrm{F}_{23}+\mathrm{F}_{24}\right| /\left(\mathrm{s}_{4}-\mathrm{s}_{3+4}\right)$

$\mathrm{F}_{23}=\left|\mathrm{F}_{23}+\mathrm{F}_{24}\right|-\mathrm{F}_{24}$

em que $F$ (g/dia) representa o fluxo determinado experimentalmente entre os compartimentos: $\mathrm{F}_{12}=$ fluxo do mineral do sangue para o trato; $\mathrm{F}_{21}=$ fluxo do trato ao sangue; $F_{23}=$ fluxo dos ossos ao sangue; $F_{32}=$ fluxo do sangue aos ossos; $\mathrm{F}_{24}=$ fluxo dos tecidos moles ao sangue; $\mathrm{F}_{42}=$ fluxo do sangue aos tecidos moles; $\mathrm{F}_{10}=$ entrada do mineral ao trato; $\mathrm{F}_{01}=$ excreção pelas fezes; $\mathrm{F}_{02}=$ excreção pela urina; $\mathrm{s}=$ atividade específica (SRA): $\mathrm{s}_{1}, \mathrm{~s}_{2}, \mathrm{~s}_{3}$ e $\mathrm{s}_{4}$ representam as atividades específicas no trato, sangue, ossos e tecidos, respectivamente; $\mathrm{t}=$ tempo em dias do início do experimento; $\mathrm{Q}(\mathrm{g})=\mathrm{o}$ total de $\mathrm{Ca}$ ou $\mathrm{P}$ no compartimento: sendo $\mathrm{Q}_{3}$ e $\mathrm{Q}_{4}$ o total do mineral nos ossos e tecidos moles.

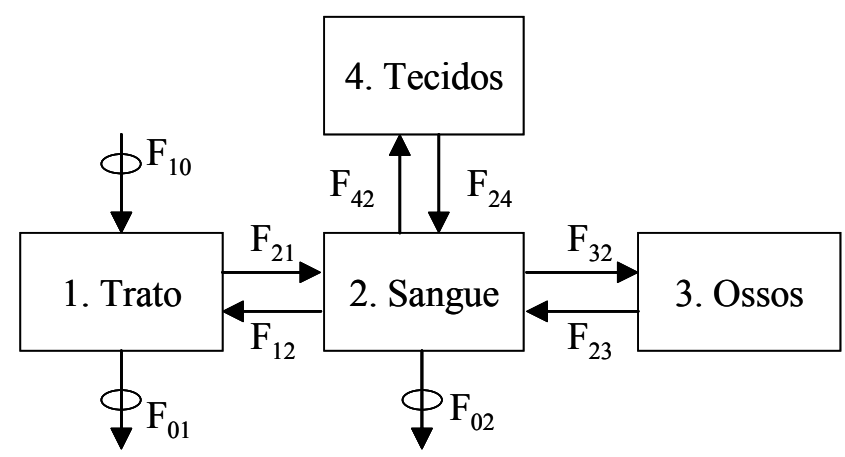

Figura 1 - Esquema do modelo de metabolismo de Ca e P, de acordo com Vitti et al. (2000). 
Os compartimentos são representados por subscritos e $\mathrm{s}_{3+4}$ é a SRA média dos compartimentos 3 e 4 (ossos e tecidos moles).

$\mathrm{O}$ total de $\mathrm{Ca}$ ou $\mathrm{P}$ endógeno nas fezes $\left(\mathrm{Fe}_{01}\right)$ foi calculado segundo a equação 8 :

$\mathrm{Fe}_{01}=\mathrm{F}_{12} \mathrm{~F}_{01} /\left(\mathrm{F}_{12}+\mathrm{F}_{10}\right)=\mathrm{s}_{1} \mathrm{~F}_{01} / \mathrm{s}_{2}$

em que $\mathrm{F}_{10}$ é o consumo de $\mathrm{Ca}$ ou $\mathrm{Pe} \mathrm{F}_{01}$ o Ca ou P nas fezes.

A absorção real de Ca ou P (Fabs) foi calculada de acordo com a equação 9:

Fabs $=\mathrm{F}_{10}-\left(\mathrm{F}_{01}-\mathrm{Fe}_{01}\right)$

Neste experimento a disponibilidade biológica foi considerada como a absorção real expressa em \% em relação ao $\mathrm{Ca}$ ou $\mathrm{P}$ consumido.

Foi utilizado o delineamento inteiramente casualizado, com três tratamentos e quatro repetições. As análises estatísticas foram realizadas utilizando-se o programa SAS (2000) e o modelo estatístico utilizado foi:

$$
\mathrm{Y}_{\mathrm{i}}=\mu+\mathrm{T}_{\mathrm{i}}+\mathrm{e}_{\mathrm{i}}
$$

em que $Y_{i}$ representa a variável dependente; $\mu$, a média geral; $\mathrm{T}_{\mathrm{i}}$, o efeito dos tratamentos $(\mathrm{i}=1,2,3) ; \mathrm{e}_{\mathrm{i}}$, o erro residual.

\section{Resultados e Discussão}

Os valores obtidos para o Ca total ingerido foram 10,$28 ; 28,08$ e 46,93 g/animal/dia, respectivamente, para os níveis 0,$15 ; 0,45$ e $0,75 \%$ de $\mathrm{Ca}$. A ingestão de $\mathrm{Ca}$ diferiu entre os tratamentos $(\mathrm{P}<0,01)$, conforme hipótese estabelecida. Segundo o NRC (1989) e Ott (1995), eqüinos em crescimento com idade de aproximadamente 12 meses devem consumir cerca de 23 a $27 \mathrm{~g}$ de cálcio/cabeça/dia. Assim, o consumo dos animais no nível 0,15\% mostrou-se deficiente em cálcio, enquanto o tratamento com $0,45 \%$ de $\mathrm{Ca}$ apresentou-se com níveis adequados e o consumo dos animais no tratamento com $0,75 \%$ de $\mathrm{Ca}$ foi superior às exigências mínimas para esta categoria (Tabela 3 ).

Os valores obtidos para o $\mathrm{P}$ total ingerido foram 13,61; 13,04 e 13,47 g/animal/dia, respectivamente, para os níveis 0,$15 ; 0,45$ e $0,75 \%$ de Ca. A ingestão de $P$ não diferiu entre os tratamentos $(\mathrm{P}>0,05)$, conforme planejado, e os níveis de $\mathrm{P}$ consumido podem ser considerados adequados. Segundo o NRC (1989) e Ott (1995), eqüinos em crescimento com idade em torno de 12 meses e peso vivo adulto previsto de
$450 \mathrm{~kg}$, com moderada velocidade de crescimento, devem consumir cerca de $13 \mathrm{~g}$ de P por cabeça/dia (Tabela 4).

As proporções Ca:P foram 0,76:1; 2,08:1 e 3,48:1, respectivamente, para 0,$15 ; 0,45$ e $0,75 \%$ de Ca.

O NRC (1989) recomendou proporção de 2:1 para potros com 12 meses, enquanto Cunha (1991) destacou que animais que receberam dietas com relação $\mathrm{Ca}: \mathrm{P}$ superior a $3: 1$ não apresentaram problemas e Lopes et al. (2003) relatou que a proporção Ca:P não deve ser inferior a 1:1.

Não houve diferenças significativas entre as concentrações de Ca no plasma $(\mathrm{P}>0,05)$ para os tratamentos. Estes resultados corroboraram os dados obtidos por Schryver et al. (1970), que, trabalhando com pôneis recebendo três diferentes níveis de Ca na dieta $(0,15 ; 0,80$ e 1,50\%), não observaram diferenças significativas na concentração de Ca no plasma para os tratamentos estudados. Esses autores obtiveram, respectivamente, valores médios de concentração de Ca no plasma de 11,70; 11,66 e 11,30 mg/dL, muito próximos aos obtidos neste trabalho e considerados normais para os eqüinos. Os autores ressaltam, ainda, que os animais responderam aos níveis de $\mathrm{Ca}$ na dieta por intermédio de diversos mecanismos, sempre no sentido da manutenção da concentração plasmática normal do elemento.

Tabela 3 - Valores médios para os parâmetros relacionados ao estudo do metabolismo e da cinética de Ca em eqüinos em crescimento

\begin{tabular}{|c|c|c|c|c|}
\hline \multirow{2}{*}{ Parâmetro (g/dia) } & \multicolumn{3}{|c|}{ Nível de Ca (\%) } & \multirow[t]{2}{*}{ EP } \\
\hline & 0,15 & 0,45 & 0,75 & \\
\hline Ingestão $\mathrm{Ca}\left(\mathrm{F}_{10}\right)$ & $10,28 \mathrm{a}$ & $28,08 \mathrm{~b}$ & $46,93 \mathrm{c}$ & 1,78 \\
\hline $\mathrm{Ca}$ fecal $\left(\mathrm{F}_{01}\right)$ & $5,30 \mathrm{a}$ & $8,15 \mathrm{a}$ & $17,40 \mathrm{~b}$ & 1,81 \\
\hline $\mathrm{Ca}$ urina $\left(\mathrm{F}_{02}\right)$ & $0,18 \mathrm{a}$ & $1,10 \mathrm{a}$ & $2,00 \mathrm{a}$ & 1,11 \\
\hline $\mathrm{Ca}$ endógeno $(\mathrm{Ca} f)$ & $0,30 \mathrm{a}$ & $1,27 \mathrm{a}$ & $4,01 \mathrm{~b}$ & 1,00 \\
\hline $\begin{array}{l}\text { Ca do sangue para } \\
\text { TGI }\left(\mathrm{F}_{12}\right)\end{array}$ & $0,62 \mathrm{a}$ & $5,72 b$ & $15,06 \mathrm{~b}$ & 4,62 \\
\hline $\begin{array}{l}\text { Ca do TGI para } \\
\text { sangue }\left(\mathrm{F}_{21}\right)\end{array}$ & $5,59 \mathrm{a}$ & $25,64 b$ & $44,58 \mathrm{c}$ & 3,88 \\
\hline Absorção real de $\mathrm{Ca}$ & $4,97 \mathrm{a}$ & $19,93 b$ & $29,53 \mathrm{c}$ & 1,48 \\
\hline $\begin{array}{l}\text { Disponibilidade } \\
\text { biológica }(\%)\end{array}$ & $48,32 \mathrm{a}$ & $62,98 b$ & $71,37 \mathrm{~b}$ & 9,10 \\
\hline $\begin{array}{l}\text { Ca do sangue } \\
\text { aos ossos }\left(\mathrm{F}_{32}\right)\end{array}$ & $286,00 \mathrm{a}$ & $303,80 \mathrm{a}$ & $414,50 \mathrm{a}$ & 118,03 \\
\hline $\begin{array}{l}\text { Ca dos ossos } \\
\text { ao sangue }\left(\mathrm{F}_{23}\right)\end{array}$ & $281,00 \mathrm{a}$ & $285,20 \mathrm{a}$ & $387,50 \mathrm{a}$ & 117,31 \\
\hline $\begin{array}{l}\text { Ca do sangue aos } \\
\text { tecidos }\left(\mathrm{F}_{42}\right)\end{array}$ & $7,84 \mathrm{a}$ & $7,61 \mathrm{a}$ & $10,01 \mathrm{a}$ & 3,5 \\
\hline $\begin{array}{l}\text { Ca dos tecidos } \\
\text { ao sangue }\left(\mathrm{F}_{24}\right)\end{array}$ & $7,73 a$ & $7,83 \mathrm{a}$ & $10,52 \mathrm{a}$ & 3,45 \\
\hline $\begin{array}{l}\text { Balanço Ca nos } \\
\text { ossos }\end{array}$ & $4,67 \mathrm{a}$ & $18,84 b$ & $26,02 \mathrm{c}$ & 1,28 \\
\hline $\begin{array}{l}\text { Balanço } \mathrm{Ca} \text { nos } \\
\text { tecidos moles }\end{array}$ & $-0,03 a$ & $0,27 \mathrm{a}$ & $0,63 \mathrm{a}$ & 0,13 \\
\hline $\mathrm{Ca}$ no plasma $(\mathrm{mg} / \mathrm{dL})$ & 11,64 & 11,94 & 11,71 & \\
\hline
\end{tabular}

EP = erro-padrão das médias.

Letras diferentes na linha indicam valores diferentes $(P<0,05)$ 
Tabela 4 - Médias das variáveis relacionadas ao metabolismo de $P$ em eqüinos em crescimento

\begin{tabular}{|c|c|c|c|c|}
\hline \multirow[t]{2}{*}{ Parâmetro (g/dia) } & \multicolumn{3}{|c|}{ Nível de $\mathrm{Ca}(\%)$} & \multirow[t]{2}{*}{ EP } \\
\hline & 0,15 & 0,45 & 0,75 & \\
\hline Ingestão $\mathrm{P}\left(\mathrm{F}_{10}\right)$ & $13,61 \mathrm{a}$ & $13,04 \mathrm{a}$ & $13,47 \mathrm{a}$ & 0,56 \\
\hline$P$ fecal $\left(\mathrm{F}_{01}\right)$ & $5,09 \mathrm{a}$ & $4,89 \mathrm{a}$ & $4,79 \mathrm{a}$ & 1,04 \\
\hline $\mathrm{P}$ urina $\left(\mathrm{F}_{02}\right)$ & $2,49 \mathrm{a}$ & $0,08 b$ & $0,25 b$ & 0,49 \\
\hline $\mathrm{P}$ endógeno $\left(\mathrm{P}_{f}\right)$ & $1,64 \mathrm{a}$ & $3,11 \mathrm{a}$ & $3,00 \mathrm{a}$ & 0,62 \\
\hline $\begin{array}{l}\mathrm{P} \text { do sangue para } \\
\text { TGI }\left(\mathrm{F}_{12}\right)\end{array}$ & $7,93 \mathrm{a}$ & $26,42 b$ & $23,99 b$ & 5,97 \\
\hline $\begin{array}{l}P \text { do TGI para } \\
\text { sangue }\left(F_{21}\right)\end{array}$ & $16,45 \mathrm{a}$ & $34,58 b$ & $32,67 b$ & 6,21 \\
\hline Absorção real de P & $10,15 \mathrm{a}$ & $11,26 \mathrm{a}$ & $11,68 \mathrm{a}$ & 0,62 \\
\hline $\begin{array}{l}\text { Disponibilidade } \\
\text { biológica }(\%)\end{array}$ & $75,53 \mathrm{a}$ & $86,32 \mathrm{a}$ & $86,54 \mathrm{a}$ & 4,53 \\
\hline $\begin{array}{l}P \text { do sangue aos } \\
\text { ossos }\left(F_{32}\right)\end{array}$ & $168,51 \mathrm{a}$ & $149,09 \mathrm{a}$ & $183,47 \mathrm{a}$ & 46,11 \\
\hline $\begin{array}{l}\mathrm{P} \text { dos ossos } \\
\text { ao sangue }\left(\mathrm{F}_{23}\right)\end{array}$ & $160,28 \mathrm{a}$ & $139,80 \mathrm{a}$ & $171,93 \mathrm{a}$ & 44,63 \\
\hline $\begin{array}{l}\text { Pdo sangue } \\
\text { aos tecidos }\left(\mathrm{F}_{42}\right)\end{array}$ & $22,08 \mathrm{a}$ & $18,40 \mathrm{a}$ & $27,49 a$ & 7,70 \\
\hline $\begin{array}{l}\mathrm{P} \text { dos tecidos } \\
\text { ao sangue }\left(\mathrm{F}_{24}\right)\end{array}$ & $24,29 a$ & $19,62 \mathrm{a}$ & $30,60 \mathrm{a}$ & 9,01 \\
\hline Balanço P nos ossos & $8,24 \mathrm{a}$ & $9,29 \mathrm{a}$ & $11,54 \mathrm{a}$ & 2,37 \\
\hline $\begin{array}{l}\text { Balanço P nos } \\
\text { tecidos moles }\end{array}$ & $-2,21 \mathrm{a}$ & $-1,21 \mathrm{a}$ & $-3,10 \mathrm{a}$ & 1,36 \\
\hline P no plasma (mg/dL) & 4,98 & 4,60 & 5,06 & \\
\hline
\end{tabular}

$\mathrm{EP}=$ erro-padrão das médias.

Letras diferentes na linha indicam valores diferentes $(P<0,05)$.

A manutenção do Ca plasmático, com mínima variação, pode ser explicada pelo mecanismo homeostático, por intermédio de um sistema hormonal constituído de paratormônio (PTH), calcitonina e da forma fisiologicamente ativa da vitamina $\mathrm{D}_{3}, 1-25$-dihidroxicolecalciferol (calcitriol, 1,25-(OH) $\left.{ }_{2} \mathrm{D}_{3}\right)$. Os níveis de Ca plasmático devem ser mantidos dentro de certo limite para manutenção normal das atividades dos tecidos nervoso e muscular. Quando há deficiência de $\mathrm{Ca}$, ocorre mobilização do Ca dos ossos. Neste estudo observou-se este fato, em particular para o tratamento $0,15 \%$, com a manutenção de Ca no plasma, mesmo havendo fluxos menores de $\mathrm{Ca}$ entre o sangue e ossos e menor balanço de Ca nos ossos.

As concentrações de $\mathrm{P}$ no plasma não diferiram significativamente $(\mathrm{P}>0,05)$ entre os tratamentos. Considerando-se os valores observados, pode-se afirmar que o nível de $\mathrm{P}$ nas dietas foi suficiente para manter valores adequados (Schryver et al., 1987). McDowell (1992) considerou que os limites normais de $\mathrm{P}$ plasmático podem variar de 4,0 a $9,0 \mathrm{mg} / \mathrm{dL}$.

Estudos com eqüinos (pôneis e cavalos) utilizando níveis de $\mathrm{P}$ na dieta variando de 0,30 a $0,70 \%$ indicaram valores de P plasmático, em média, de $4,50 \mathrm{mg} / \mathrm{dL}$, valor muito próximo aos obtidos neste trabalho (Tabela 4) usando semelhante nível de P na dieta (Schryver et al., 1987; Argenzio et al., 1973; Hintz et al., 1972 ).
Não houve diferença significativa para os valores de $\mathrm{Ca}$ na urina $\left(\mathrm{F}_{02}\right)$, no entanto, observou-se grande variação, com o nível de $0,45 \%$ Ca apresentando valor acima de dez vezes superior ao de $0,15 \%$. Estes resultados podem indicar que, quando os animais ingerem excesso de $\mathrm{Ca}, \mathrm{o}$ sistema renal pode ser a principal rota de excreção. Van Doorn et al. (2004) observaram, em pôneis, incremento significativo na excreção de Ca via urina, com aumento do consumo deste mineral.

Os valores obtidos para o $\mathrm{P}$ excretado na urina foram 2,$49 ; 0,08$ e $0,25 \mathrm{~g} /$ dia para os níveis 0,$15 ; 0,45$ e $0,75 \%$ respectivamente. Para o nível $0,15 \%$, os valores foram mais elevados $(\mathrm{P}<0,01)$. A excreção urinária de $\mathrm{P}$ nos eqüinos é baixa $(<2 \%)$, entretanto, para o tratamento $0,15 \%$, observa-se que a excreção correspondeu a aproximadamente $18 \%$ do $\mathrm{P}$ consumido, tendo sido provavelmente influenciada pela falta de Ca na dieta, demonstrando a importância da relação Ca:P na dieta. Embora a excreção urinária de P para este tratamento tenha sido elevada $(2,49 \mathrm{~g} / \mathrm{animal} /$ dia), nenhum dos tratamentos foi deficitário neste elemento. Experimentos com pôneis mostraram que dietas com baixo Ca e alto $\mathrm{P}$ causaram maior excreção urinária de $\mathrm{P}(90 \%$ do $\mathrm{P}$ absorvido) do que dietas com alto Ca e P (40-50\% do P absorvido) (Kichura et al., 1983). Deve-se considerar ainda o efeito do hormônio da paratireóide, que em dietas deficientes em $\mathrm{Ca}$ atua no sentido de manter os níveis plasmáticos deste mineral e pode acarretar maior excreção de $\mathrm{P}$ pelos rins.

Há poucas informações quanto aos níveis de excreção renal de $\mathrm{P}$ em monogástricos. Estudos com animais monogástricos (Kolb, 1979; Fernández, 1995) indicam que a excreção renal de P não é principal via de excreção de P. Entretanto, cavalos e suínos podem excretar grandes quantidades de $\mathrm{P}$ via urina, quando a ingestão de $\mathrm{P}$ for alta, o que leva os rins a terem importante função na manutenção da homeostase do P.

O elevado valor do $\mathrm{P}$ excretado na urina para o nível 0,15\% em relação ao $\mathrm{P}$ consumido, compara-se a dados indicados por Schryver et al. (1971) e Argenzio et al. (1973), que, utilizando dietas com altos níveis de $\mathrm{P}$ consumido (200 mg de P/kgPV/dia), relataram que a excreção renal de $\mathrm{P}$ atingiu valores entre 20 e $30 \%$ do $\mathrm{P}$ consumido. Esses autores concluíram que a elevação na excreção urinária indica um ajuste renal na eliminação do P. Cunha (1991) também demonstrou que o excesso de $P$ consumido resulta em maior excreção urinária do elemento. Neste experimento, o $P$ consumido correspondeu a $62 \mathrm{mg} / \mathrm{kg}$ PV/dia, valor bem abaixo daquele citado acima, sugerindo que outros fatores, como o teor de Ca na dieta, influenciaram a excreção de $\mathrm{P}$. Observa-se também que os valores de P endógeno fecal 
foram menores para o tratamento $0,15 \%$, indicando mudança na rota de excreção do $\mathrm{P}$, por efeito hormonal, conforme já citado. Van Doorn et al. (2004) observaram menor excreção renal de $\mathrm{P}$ em pôneis que receberam dietas com níveis moderados e altos de $\mathrm{Ca}$.

A excreção de $\mathrm{Ca}$ nas fezes $\left(\mathrm{F}_{10}\right)$ foi influenciada $(\mathrm{P}<0,01)$ pelos tratamentos, sendo maior para o nível $0,75 \%$. Hintz \& Schryver (1972), em estudo com pôneis recebendo dieta com 0,30 a $0,44 \%$ de Ca, observaram excreção fecal de 6 a 7,5 g Ca/animal/dia; os valores obtidos neste trabalho corroboram esses dados.

Embora a excreção fecal de Ca nos animais com 0,75\% de Ca seja elevada (17,40 g/dia), quando se faz a relação entre o $\mathrm{Ca}$ ingerido e o $\mathrm{Ca}$ excretado, nota-se que o $\mathrm{Ca}$ excretado nas fezes correspondeu a 51,56; 29,02 e 37,08\% do Ca consumido, respectivamente, para os níveis 0,15 ; 0,45 e $0,75 \%$. O valor alto de Ca nas fezes para o $0,15 \%$ pode ser resultado da relação Ca:P na dieta, seja, 0,76:1, 2,08:1 e $3,48: 1$, respectivamente para os níveis 0,$15 ; 0,45$ e $0,75 \%$. A relação Ca:P menor que 1 , no tratamento com $0,15 \%$ de Ca, pode ter influenciada a absorção de $\mathrm{Ca}$, o que fica evidenciado nos valores observados para o Ca endógeno, absorção real e disponibilidade. $\mathrm{O}$ excesso de $\mathrm{P}$ em relação ao $\mathrm{Ca}$ tem influência na utilização de $\mathrm{Ca}$, maior que o reverso, seja o excesso de Ca em relação ao P (Hintz, 1990). Van Doorn et al. (2004) também observaram que o fornecimento de excessivas quantidades de $\mathrm{P}$ em relação ao $\mathrm{Ca}$ causou efeito depressivo na digestibilidade aparente de $\mathrm{Ca}$.

O P total excretado nas fezes não foi afetado pelos níveis de cálcio $(\mathrm{P}>0,05)$. Neste trabalho, o $\mathrm{P}$ excretado nas fezes correspondeu, em média, a $36,47 \%$ do $P$ consumido. Estes valores são mais baixos que aqueles encontrados por Furtado et al. (2000) e Oliveira (1999), que, trabalhando com eqüinos em crescimento, obtiveram, respectivamente, médias percentuais de 79,8 e 59,57\%.

Considerando-se que a principal via de eliminação do P é a excreção fecal (Hintz \& Schryver, 1972; Furtado et al., 2000; Van Doorn et al., 2004), os valores relativamente baixos encontrados neste trabalho podem ser explicados pela elevada absorção intestinal do mineral.

Os valores médios de perda endógena fecal para os níveis 0,$15 ; 0,45$ e $0,75 \%$ de Ca foram, respectivamente, 1,64 ; 3,11 e 3,00 g/animal/dia. Não houve diferença significativa entre os tratamentos $(\mathrm{P}>0,05)$, apesar de o valor médio para $0,15 \%$ ser numericamente mais baixo. Para $0,15 \%$ de Ca, o $\mathrm{P}$ endógeno representou $32 \%$ em relação ao $P$ total excretado e para os tratamentos 0,45 e $0,75 \%$ os valores foram, em média, 63\%. Furtado et al. (2000) e Oliveira (1999) relataram, respectivamente, valores médios de 27,9 e $14,55 \%$ do $\mathrm{P}$ endógeno em relação ao $\mathrm{P}$ total excretado nas fezes. Cymbaluck et al. (1989), utilizando potros em crescimento e nível de $P$ consumido de $160 \mathrm{mg} / \mathrm{kg}$ PV/dia, obtiveram valor de P endógeno fecal de $17,9 \mathrm{mg} / \mathrm{kg}$ PV/dia, o que representou $15,6 \%$ do $\mathrm{P}$ total excretado. Os valores de $\mathrm{P}$ endógeno obtidos neste trabalho foram maiores que os citados na literatura. O P endógeno representa o $\mathrm{P}$ resultante do metabolismo do animal (P reciclado salivar, restos celulares), que já foi utilizado nos processos metabólicos, refletindo na absorção e disponibilidade. Isto pode ser verificado pelos elevados valores de absorção real (média 11,03 g/dia) e disponibilidade biológica (média de $83 \%$ ) obtidos neste trabalho.

Os resultados de disponibilidade biológica de $\mathrm{Ca}$ (Tabela 3) para os animais nos tratamentos $0,45 \%(62,98 \%)$ e $0,75 \%$ (71,37\%) estão em concordância com Hintz \& Schryver(1972) e Hintz (1990), que obtiveram, para pôneis que receberam diferentes fontes de $\mathrm{Ca}$, valor médio de $70,2 \%$. No entanto, para o tratamento com menor relação $\mathrm{Ca}: \mathrm{P}(0,15 \%)$, a disponibilidade biológica do Ca foi menor $(48,32 \%)$.

Os fluxos de $\mathrm{Ca}$ do sangue para o trato gastrintestinal $\left(\mathrm{F}_{12}\right)$ e vice-versa $\left(\mathrm{F}_{21}\right)$ foram menores para $0,15 \%(\mathrm{P}<0,01)$, aumentando com a quantidade de $\mathrm{Ca}$ ingerida. A diferença entre $\mathrm{F}_{21}$ e $\mathrm{F}_{12}$ resulta na absorção real, cujos valores foram 4,$97 ; 19,93$ e 29,53 g/dia, respectivamente, para os níveis 0,15 ; 0,45 e $0,75 \%$ de $\mathrm{Ca}(\mathrm{P}<0,01)$. Em eqüinos, o desequilíbrio nutricional na dieta e na homeostase corporal freqüentemente ligado aos minerais é a excessiva ingestão de $\mathrm{P}$ ou a insuficiente quantidade de $\mathrm{Ca}$ na dieta.

No tocante à mobilização $\left(\mathrm{F}_{23}\right)$ e absorção $\left(\mathrm{F}_{32}\right)$ de Ca nos ossos, não se verificou diferença entre os tratamentos, o que se deve provavelmente às variações nos dados obtidos, decorrentes provavelmente da amostragem dos ossos. Embora todo cuidado tenha sido tomado na separação de partes semelhantes dos ossos, em todos os animais, algumas variações podem ter ocorrido. Mesmo assim, não se pode ignorar que houve maior mobilização de Ca para os ossos, o que refletiu no balanço de $\mathrm{Ca}\left(\mathrm{F}_{32}-\mathrm{F}_{23}\right)$, resultando em aumentos significativos $(\mathrm{P}<0,01)$ para os níveis $0,45 \%$ (18,84 g/dia) e $0,75 \%$ (26,02 g/dia) em relação à menor quantidade de $\mathrm{Ca}$ ingerida $(0,15 \%)(4,67 \mathrm{~g} / \mathrm{dia})$. Estes resultados mostram que há maior deposição do $\mathrm{Ca}$ nos ossos, quando níveis considerados adequados ou acima das exigências são oferecidos aos animais. Deve ser lembrado que os resultados deste foram obtidos com animais mantidos em gaiolas, sem exercícios físicos, devendo interpretá-los com cautela. Animais jovens, em exercício moderado, podem perder pequenas quantidades 
de $\mathrm{Ca}$ dos ossos, mas o inverso pode ocorrer quando mantidos sem exercício (Schryver \& Hintz, 1972).

A passagem de $\mathrm{Ca}$ do sangue para tecidos moles $\left(\mathrm{F}_{42}\right)$ e dos tecidos para o sangue $\left(\mathrm{F}_{24}\right)$ não foi diferente $(\mathrm{P}>0,05)$ para os tratamentos. O balanço nos tecidos $\left(\mathrm{F}_{42}-\mathrm{F}_{24}\right)$ também não apresentou diferenças para os tratamentos, embora se observe balanço negativo para $0,15 \%$ de $\mathrm{Ca}$, sugerindo que, para manter níveis adequados de $\mathrm{Ca}$ no sangue, ocorre também mobilização do Ca presente nos tecidos moles.

As trocas de $\mathrm{P}$ entre sangue e trato $\left(\mathrm{F}_{12}\right.$ e $\left.\mathrm{F}_{21}\right)$, observadas pelo modelo de metabolismo de $\mathrm{P}$, foram inferiores para o tratamento com $0,15 \%$ de $\mathrm{Ca}$. Entretanto, quando se faz a diferença entre esses fluxos $\left(\mathrm{F}_{21}-\mathrm{F}_{12}\right)$, para os três tratamentos, os valores foram de 8,$52 ; 8,16$ e 8,68 g, mostrando que a proporção $\mathrm{Ca}: \mathrm{P}$ não afetou o balanço trato-sangue. Isto também explicaria porque o $\mathrm{P}$ endógeno não foi significativamente diferente entre os tratamentos. Como o consumo de $\mathrm{P}$ foi semelhante entre os tratamentos e a quantidade líquida de $\mathrm{P}$ que voltou ao trato foi semelhante, o P endógeno também não foi diferente $(\mathrm{P}>0,05)$.

Os demais parâmetros relacionados ao metabolismo de P não foram afetados pelos níveis de $\mathrm{Ca}$ (Tabela 3). Os dados do balanço de $\mathrm{P}$ nos ossos indicam que houve deposição média óssea de 9,69 g/dia. Portanto, a quantidade de $\mathrm{P}$ fornecida diariamente aos animais $(13,37 \mathrm{~g} / \mathrm{dia})$ foi suficiente para os animais experimentais. Furtado et al. (2000), trabalhando com potros de 19 meses de idade, da raça Brasileiro de Hipismo, obtiveram exigência de 14,75 g de P/ dia, inferior à recomendação do NRC (1989), de 16 g de P/dia.

$\mathrm{O}$ balanço de $\mathrm{P}$ nos tecidos foi negativo para todos os tratamentos. A causa desta ocorrência não está clara. Provavelmente, o $\mathrm{P}$ retirado dos tecidos foi utilizado para a manutenção do P no plasma (4,98; 4,60 e 5,06 mg/dL, para os níveis 0,$15 ; 0,45$ e $0,75 \%$,respectivamente) e também para a deposição óssea.

\section{Conclusões}

Foi possível, por intermédio de modelos matemáticos determinístico e compartimental associados ao uso da técnica de diluição isotópica, com a aplicação de ${ }^{45} \mathrm{Ca}$ e ${ }^{32} \mathrm{P}$, validar estes métodos pela verificação do metabolismo e da cinética do $\mathrm{Ca}$, em potros, ao constatar-se que as diferentes proporções $\mathrm{Ca}: \mathrm{P}$ na dieta realmente são determinantes sobre a excreção, retenção e absorção de cálcio.

\section{Literatura Citada}

ARGENZIO, R.A.; LOWE, J.E.; HINTZ, H.F. et al. Calcium and phosphorus homeostasis in horse. Journal of Nutrition, v.104, n.1, p.18-24, 1974.
ASSOCIATION OF OFFICIAL ANALYTICAL CHEMISTS - AOAC. Official methods of analysis. 13.ed. Washington, D.C.: 1980. 1018p

CAVERZASIO, J.; BONJOUR, J.P.; FLEISCH, H. Tubular handling of $\mathrm{P}_{\mathrm{i}}$ in young growing and adults rats. American Journal of Physiology - Renal Physiology, v.242, n.6, p.705-710, 1982

CHAPUIS-LARDY, L.; FIORINI, J.; TOTH, J.D. et al. Phosphorus concentrations in dairy feces: variability and affecting factors Journal of Dairy Science, v.87,v.12, p.4334-4341, 2004.

CROMWELL, G.L. Phytase appears to reduce phosphorus in feed manure. Feedstuffs, v.63, n.28, p.41, 1991.

CUNHA, T.J. Horse feeding and nutrition. 2.ed. San Diego: Academic Press, 1991. 445p.

CUPÁK, M.; PROCHAZKA, Z.; JAMBO, R.V. Utilization of phosphorus in pigs after endogenous phosphorus determination by means of ${ }^{32} \mathrm{P}$. Acta Veterinaria B., v.41, p.257-262, 1972.

CYMBALUCK, N.F.; CHRISTISON, G.L.; LEACH, D.H. Nutrient utilization by limit and ad libidum fed growing horses. Journal of Animal Science, v.67, n.2, p.414-425, 1989.

DIAS, R.S.; KEBREAB, E.; VITTI, D.M.S.S. et al. A revised model for studying phosphorus and calcium kinetics in growing sheep. Journal of Animal Science, v.84, p.2787-2794, 2006.

FERNANDEZ, J.A. Calcium and phosphorus metabolism in growing pigs. III. A model resolution. Livestock Production Science, v.41, n.1, p.255-2261, 1995.

FISKE, C.H.; SUBBAROW, Y. The colorimetric determination of phosphorus. Journal Biology Chemistry, v.66, n.2, p.375400,1925

FURTADO C.E.; TOSI, H.; VITTI, D.M.S.S. Gaiola de metabolismo para eqüinos. Acta Scientiarum, v.22, n.3, p.813-816, 2000.

HINTZ, H.F. Factors affecting nutrient availability in the horse. In: GEORGIA NUTRITION CONFERENCE FOR THE FEED INDUSTRY, 1990, Atlanta. Proceedings... Atlanta: 1990. p.181-193.

HINTZ, H.F.; SCHRYVER, H.F. Availability of ponies of calcium and phosphorus from various supplements. Journal of Animal Science, v.34, n.4, p.979-980, 1972.

INTERNATIONAL ATOMIC ENERGY AGENCY - IAEA Laboratory training manual on the use of nuclear techniques in animal research. Vienna: IAEA, 1979. 299p. (Technical Report Series, 193).

KOLB, E. Fisiologia veterinária. 2.ed. Zaragoza: Acribia, 1979. $1115 \mathrm{p}$.

LOPES, J.B.; FURTADO, C.; VITTI, D.M.S.S. et al. Metabolismo de fósforo em eqüinos. 1. Avaliação dietética de diferentes fontes de fósforo. Revista Brasileira de Zootecnia, v.32, n.6, p.1339-1347, 2003

McDOWELL, L.R. Mineral in animal and human nutrition San Diego: Academic Press, 1992. 524p.

MORSE, D.; HEAD,H.H.; WILLOX,C.J. et al. Effects of concentration of dietary phosphorus on amount and route of excretion. Journal of Dairy Science, v.75, n.11, p.3039-3949, 1992.

NATIONAL RESEARCH COUNCIL - NRC. Nutrient requirements of horses. 5.ed. Washington, D.C: National Academy of Sciences, 1989. 100p.

OLIVEIRA, A.A.M.A. Avaliação da disponibilidade biológica do fósforo em diferentes dietas para potros em crescimento. Jaboticabal: Universidade Estadual Paulista, 123p. 1999. Tese (Doutorado em Zootecnia) - Universidade Estadual Paulista, 1999.

OTT, E.A. Dietary nutrient allowances for horses. Feedstuffs, v.64, n.29, p.77-80, 1995

SALVIANO, L.M.C.; VITTI, D.M.S.S. Influência da proporção de cálcio e fósforo na dieta, nas perdas endógenas e na absorção de fósforo em ovinos. Pesquisa Agropecuária Brasileira, v.33, n.3, p.349-355, 1998.

SARRUGE, J.R.; HAAG, H.P. Análises químicas em plantas. Piracicaba: ESALQ/USP, 1974. 56p.

SCHRYVER, H.F.; GRAIG, P.H.; HINTS, H.F. Calcium metabolism in ponies fed varying levels of calcium. Journal of Nutrition, v.100, n.5, p.955-964, 1970. 
SCHRYVER, H.F.; HINTS, H.F.; GRAIG, P.H. Phosphorus metabolism in ponies fed varyng levels of phosphorus. Journal of Nutrition, v.101, n.5, p.1257-1264, 1971.

SCHRYVER, H.F.; PARKER, M.T.; DANILUK, P.D. et al. Salt consumption and the effect of salt on mineral metabolism in horses. Cornell Veterinary, v.77, n.1, p.122-131, 1987.

STATISTICAL ANALYSIS SYSTEM - SAS. The SAS system for windows. Release 8.01. Cary: 2000. (CD-ROM).

TAMIM, N.M.; ANGEL, R. Phytate phosphorus hydrolysis as influenced by dietary calcium and micro-mineral source in broiler diets. Journal of Agricultural and Food Chemistry, v.51, n.16, p.4687-4693, 2003.
Van DOORN, D.A.; van der SPEK, M.E.; EVERTS, H. et al. The influence of calcium intake on phosphorus digestibility in mature ponies. Journal of Animal Physiology and Animal Nutrition, v.88, n.11, p.412-418, 2004.

VITTI, D.M.S.S.; KEBREAB, E.; LOPES, J.B. et al. A kinetic model of phosphorus metabolism in growing goats. Journal of Animal Science, v.78, n.10, p.2706-2712, 2000

ZAGATTO, E.A.G.; KRUG, F.J.; BERGAMIN FILHO, H. et al. Merzing in flow injection analysis. Part 2. Determination of calcium, magnesium and posstassium in plant material by flow injection atomic and flame emission spectrometry. Analytica Chimica Acta, v.104, n.2, p.279-284, 1979. 